\title{
TRABALHO DE CAMPO EM PESQUISA QUANTITATIVA NA PERSPECTIVA DOS ENTREVISTADORES: ANÁLISE QUALITATIVA*
}

\author{
Ana Lúcia Cardoso Kirchhof ${ }^{1}$, Verônica de Azevedo Mazza², Ana Maria Cosvoski Alexandre², \\ Leila Maria Mansano Sarquis ${ }^{4}$
}

\begin{abstract}
RESUMO: Devido à importância do trabalho de campo em uma pesquisa, objetivou-se analisá-lo a fim de destacar os procedimentos necessários à diminuição de perdas e recusas na coleta de informações. Trata-se de uma pesquisa descritiva com abordagem qualitativa. Foram sujeitos do estudo 22 mestrandos do Programa de Pós-Graduação em Enfermagem da Universidade Federal do Paraná, que realizaram entrevistas e responderam a instrumentos com perguntas abertas, em agosto de 2009. A análise dos dados foi realizada mediante pré-análise, tratamento e categorização. Foram destacadas as categorias estratégias para tornar o processo de entrada em campo mais eficiente, a importância da mediação do supervisor no processo e os encontros sistemáticos para dirimir dúvidas e encaminhar pendências. Destaca-se ainda a importância de aprofundar e discutir os aspectos metodológicos de uma pesquisa a fim de qualificar esse processo.
\end{abstract}

PALAVRAS-CHAVE: Coleta de dados; Pessoal de saúde; Pesquisa; Entrevistas como assunto.

\section{FIELD WORK IN QUANTITATIVE RESEARCH IN THE PERSPECTIVE OF INTERVIEWERS: QUALITATIVE ANALYSIS}

ABSTRACT: The authors aimed to analyze field work, due to its importance in research, so as to highlight the procedures necessary for reducing losses and refusals during information collection. The research is descriptive, with a qualitative approach. 22 students on the Master's course from the Post-graduate Nursing Program of the Federal University of Paraná took part in the study in August 2009, carrying out interviews and answering instruments with open questions. Data analysis was undertaken through pre-analysis, treatment and categorization. The categories singled out were: strategies for making entry into the field more efficient, the importance of the supervisor's mediation in the process, and systematic meetings to settle doubts and resolve backlogs. Further, the importance is emphasized of deepening and discussing the methodological aspects of research so as to qualify this process.

KEYWORDS: Data collection; Health personnel; Research; Interviews with subject.

\section{TRABAJO DE CAMPO EN INVESTIGACIÓN CUANTITATIVA EN LA PERSPECTIVA DE LOS ENTREVISTADORES: ANÁLISIS CUALITATIVO}

RESUMEN: A causa de la importancia del trabajo de campo en una investigación, el objetivo fue analizarlo a fin de destacar los procedimientos necesarios a la reducción de pérdidas y recusas para recoger informaciones. Esta es una investigación descriptiva con abordaje cualitativo. Fueron sujetos del estudio 22 mestrandos del Programa de Posgrado en enfermería de la Universidad Federal de Paraná, que realizaron entrevistas y contestaron a instrumentos con preguntas abiertas, en agosto de 2009. El análisis de los datos fue realizado por medio de preanálisis, tratamiento y categorización. Fueron destacadas las categorías estrategias para volver el proceso de entrada en campo más eficiente, la importancia de la mediación del supervisor en proceso y los encuentros sistemáticos para dirimir dudas y encaminar pendencias. Se destaca todavía la importancia de profundizar y discutir los aspectos metodológicos de una investigación a fin de cualificar ese proceso.

PALABRAS CLAVE: Proceso de recoger datos; Personal de salud; Investigación; Entrevistas como asunto.

\footnotetext{
* Pesquisa financiada pelo CNPq, Edital MCT/CNPq 14/2009, processo n. ${ }^{\circ}$ 472346/2009-1.

${ }^{1}$ Enfermeira. Doutora em Enfermagem. Professora Visitante do Programa de Pós-Graduação em Enfermagem da Universidade Federal do Paraná - PPGENF UFPR.

${ }^{2}$ Enfermeira. Doutora em Enfermagem. Professora do Departamento de Enfermagem e do PPGENF UFPR. Líder do Grupo de Estudo da Família, Saúde e Desenvolvimento.

${ }^{3}$ Enfermeira da Prefeitura Municipal de Curitiba. Mestre em Enfermagem.

${ }^{4}$ Enfermeira. Doutora em Enfermagem. Professora do Departamento e do PPGENF UFPR. Membro do Grupo de Pesquisa Multiprofissional em Saúde do Adulto.
}

Autor correspondente:

Ana Lúcia Cardoso Kirchhof

Universidade Federal do Paraná

Rua Vereador Ramon Filomeno, 183 - 88034-495-Florianopolis-SC-Brasil

E-mail: kirchhof@terra.com.br
Recebido: 03/12/2011

Aprovado: 14/03/2012

Cogitare Enferm. 2012 Abr/Jun; 17(2):284-9 


\section{INTRODUÇÃO}

Trabalhar com pesquisa é uma atividade desafiadora em vários aspectos. $\mathrm{O}$ trabalho de campo e a coleta de informações primárias, discutida neste estudo, é obtida por meio de pesquisa de campo, considerada de fundamental importância, tendo recebido atenção especial em publicações ${ }^{(1-5)}$. Na pesquisa quantitativa, o pesquisador define previamente parâmetros de qualidade e quantidade que não poderão ser alterados, sem que haja prejuízo para os resultados esperados. Sendo assim, o estudo e o aprimoramento desse processo são aspectos fundamentais para produção de pesquisas de qualidade, haja vista que neste tipo de abordagem não há muita flexibilidade para alterar o planejamento previsto para a coleta de informações ${ }^{(6)}$. Principalmente, quando envolve vários sujeitos com diferentes formas de participação, como entrevistadores, supervisores da coleta de dados, representantes da instituição pesquisada e sujeitos da pesquisa.

Este estudo é derivado de um macro-projeto que buscou analisar a saúde de trabalhadores de enfermagem com vínculo municipal na Atenção Básica de Saúde em uma cidade do Estado do Paraná. Previa-se realizar 1.800 entrevistas em um período de trabalho de campo de três meses. Este estudo será denominado doravante de pesquisa primária.

Em estudos de abordagem quantitativa, que prevêem um grande número de sujeitos, é preciso considerar o período da coleta de informações, pois ao se alongar, promove uma possibilidade maior de vieses, visto que a vida tem sua dinâmica própria e que as pessoas e instituições mudam ${ }^{(4)} \mathrm{em}$ função de fatores climáticos, como o frio e o calor, de políticas organizacionais, como mudanças na gerência, na divisão e organização do trabalho e também da conjuntura local e regional, como mudanças de filosofia, política, convênios e acordos; aspectos que se traduzem em reflexos nos sujeitos pesquisados ${ }^{(7)}$, especialmente naqueles que ocupam empregos públicos, como na saúde municipal.

Para dar conta desse processo, foi criada, na pesquisa primária, uma estrutura de suporte com a finalidade de troca de experiências entre entrevistadores, supervisores e coordenador do projeto de pesquisa, garantindo a homogeneidade e fidelidade na coleta de informações. Para tanto, desenvolveu-se um projeto paralelo, apresentado nesse trabalho como pesquisa secundária, cujo trabalho de campo, pela exigência de maior complexidade na sua organização, passou a ser objeto deste estudo. O que se pretendeu foi voltar o olhar, com outra investigação, ao trabalho de campo realizado, aos procedimentos usados para organizar e realizar a coleta de informações, no sentido de avaliar tanto os que foram mais adequados, como os menos adequados e porque assim ocorreu. Nossa questão orientadora foi: quais foram as estratégias usadas pelos entrevistadores para realizar a coleta das informações? Quais foram suas falhas? E, finalmente, como podemos aprimorá-lo, de modo a acrescentar valor a esse processo e contribuir com outros pesquisadores no planejamento dessa importante etapa da pesquisa?

Portanto, tem-se como objetivo analisar o processo de trabalho de campo, realizado em uma pesquisa de abordagem quantitativa, a fim de destacar os procedimentos necessários à diminuição de perdas e recusas na coleta de informações.

\section{MÉTODO}

Pesquisa descritiva com abordagem qualitativa, cuja coleta de informações ocorreu em agosto de 2009. Teve como objeto de estudo o trabalho de campo de uma pesquisa primária de abordagem quantitativa, com trabalhadores da Atenção Básica de Saúde em um município do Estado do Paraná.

Na pesquisa primária foram capacitados previamente 50 entrevistadores, sendo 23 mestrandos e 27 alunos de graduação. A capacitação foi desenvolvida em uma tarde, por meio da exposição do projeto de pesquisa, da entrega e leitura do Manual do Entrevistador e do instrumento a ser aplicado. Também foi realizada a dramatização de uma entrevista por dois entrevistadores, com a finalidade de observar posturas, atitudes, entonação de voz, indução de respostas, e melhor desempenho para questões que poderiam despertar constrangimento no entrevistado. Foram introduzidos no processo os supervisores, professores do programa de pós-graduação e da graduação, que foram responsáveis por um grupo de até 10 entrevistadores. Os responsáveis seguiram os seguintes passos: reunião semanalmente, entregando e recebendo os instrumentos, revisando-os quanto ao correto e adequado preenchimento, ouvindo e encaminhando suas dificuldades ${ }^{(8)}$. Os supervisores foram os mediadores no processo, no entanto, para esta pesquisa secundária tomaremos o processo de coleta de dados realizada apenas pelos entrevistadores mestrandos que aceitaram livremente participar do estudo.

Neste caso, o pesquisador foi aquele que concebeu o desenho do macro-projeto e coordenou os processos que permearam este trabalho. Coube ao pesquisador 
o processo de negociação e proposição da pesquisa na Secretaria Municipal de Saúde do município que forneceu os insumos para o macro-projeto: os sujeitos e seus locais de trabalho. À Secretaria Municipal de Saúde coube informar as Unidades Básicas de Saúde (UBS) da pesquisa. Ao entrevistador coube fazer contato com as UBS sob sua responsabilidade e a coleta dos dados dos trabalhadores já selecionados, por meio de uma nominata previamente construída. Para equilibrar o processo, foi distribuído um número semelhante de trabalhadores por entrevistador. Isso, por vezes, o colocou em mais de uma UBS para coleta.

Os sujeitos da pesquisa secundária foram 19 entrevistadores, mestrandos do Programa de Pós-Graduação em Enfermagem da Universidade Federal do Paraná, que assinaram o Termo de Consentimento Livre e Esclarecido.

A coleta de informações consistiu de dois instrumentos com perguntas abertas. Um voltado às negociações efetivadas pelo entrevistador e outro direcionado ao processo de supervisão. O primeiro instrumento perguntava: 1) Com quais sujeitos do campo de coleta você teve que negociar para fazer as entrevistas? 2) Que estratégias (discursivas ou não) de sedução foram usadas por você? 3) Descreva estas estratégias. 4) Quais destas estratégias você considerou mais produtivas para atingir seu objetivo? 5) Ao término da coleta de dados, caso você fosse coordenar essa pesquisa, ou fosse refazê-la, o que modificaria no processo da supervisão, entrada em campo e coleta dos dados?

O segundo instrumento continha as questões: 1) Quais foram as contribuições dos supervisores para sua trajetória nesse período de coleta de informações? 2) Quais foram as dificuldades dos professores supervisores e quais suas sugestões para aprimorar essa atividade?

A análise dos dados foi realizada mediante a proposta operativa para análise de dados qualitativos, estruturada a partir da pré-análise, tratamento e categorização dos resultados ${ }^{(9)}$, de modo a abranger a totalidade de significados ao tema em questão.

Este estudo foi submetido ao Comitê de Ética em Pesquisa do Setor de Ciências da Saúde da Universidade Federal do Paraná, processo $n^{\circ}$ 639.176.08.10, CAAE n ${ }^{\circ}$ 0067.0.091.091-08.

\section{RESULTADOS}

Os dados obtidos com o primeiro instrumento permitiram expressar o processo de negociação dos entrevistadores, na categoria Estratégias para tornar o processo de entrada em campo mais eficiente.
Para etapa inicial do processo de negociação foi referida a Autoridade Sanitária Local (ASL) como sujeito-chave para negociar a entrada em campo. No entanto, as enfermeiras responsáveis pelo turno de trabalho também foram referidas pelos entrevistadores como elementos-chave, ajudando, inclusive, na organização dos trabalhadores e na sua liberação dos postos de trabalho. Registrou-se, ainda, a dificuldade com as ASL, pois muitas alegaram não estarem cientes da realização da pesquisa, dificultando e atrasando o processo. Essa situação foi geradora de morosidade, desgaste físico e emocional, exigindo do entrevistador persistência e compromisso.

Foram referidas estratégias de sedução como: diálogo com os sujeitos da pesquisa e ASL, contato pessoal com a ASL, oferecer disponibilidade de tempo e adequação às normas da UBS, sensibilização dos profissionais em reunião de equipe e oferecer um "agrado" culinário nas entrevistas, contato telefônico prévio e boa apresentação pessoal. Outras estratégias foram destacadas, tais como: quando o tempo disponibilizado pela ASL foi muito pequeno em relação à quantidade de entrevistas a serem realizadas, a entrevistadora negociou com outra entrevistadora para irem juntas, assim, poderiam fazer mais entrevistas em menos tempo. Um intensivo de coleta na UBS também se mostrou uma estratégia profícua, visto que o entrevistador se tornou conhecido pela assiduidade no local, promovendo mais momentos de interação com o trabalhador. Igualmente interessantes foram as estratégias que ofereceram um "mimo", desvelo ou cortesia ao trabalhador, em troca da disponibilidade para a entrevista.

Outras estratégias usadas pelos entrevistadores foram: mostrar aos sujeitos a importância e relevância da pesquisa na sua vida profissional, o estabelecimento de relações de confiança com a equipe e com a ASL, não criando conflitos em momentos de dificuldade, mostrar-se disponível e consciente das normativas da UBS, uma "boa e simpática" apresentação pessoal e, sobretudo, a coleta no horário de trabalho.

Ainda, sugeriram que se oportunizasse aos sujeitos da pesquisa, em reuniões das equipes em cada UBS, uma explicação dos objetivos da pesquisa e do instrumento usado, de modo a facilitar a colaboração.

As sugestões dos entrevistadores ao processo de pesquisa foram as seguintes: maior aproximação dos entrevistadores com o tema da pesquisa, criando afinidade e vínculo com os objetivos propostos; contato com todas as ASL para entrada em campo; pagamento pelas entrevistas e ressarcimento das despesas com 
deslocamento para os campos de coleta; aumento do tempo de capacitação, abrangendo duas tardes, em vez de uma; ir às reuniões das equipes nas UBS, e explicar a pesquisa; ir a cada UBS verificar as condições e traçar estratégias para a coleta.

Os resultados relativos ao segundo instrumento procuraram aprofundar o conhecimento sobre o processo de supervisão e se organizaram nas categorias: Importância de mediação do supervisor no processo e Encontros sistemáticos para dirimir dúvidas e encaminhar pendências. Ressaltaram que o supervisor serviu de elo entre o pesquisador e o entrevistador, contribuindo para a organização do processo, tendo sido referência para eventuais dúvidas. Os entrevistadores mencionaram tanto a disponibilidade dos supervisores como a falta de tempo dos mesmos. Sugeriam, ainda, trabalhar com os supervisores, de modo que eles agendassem encontros com os entrevistadores para entrega de instrumentos, suprimir dúvidas, encaminhar dificuldades; e reuniões periódicas com os supervisores para discutir o andamento da coleta. Um entrevistador elogiou a capacitação inicial e o manual recebido como orientador de todo o processo. As ressalvas ao processo de supervisão se ativeram a diferenças nas informações prestadas entre os supervisores e à pesquisadora, à falta de um encontro sistemático para entrega dos instrumentos, ao encaminhamento de pendências e dúvidas e ao acúmulo de atividades executadas pelos supervisores.

\section{DISCUSSÃO}

De modo geral, os entrevistadores referiram dificuldade em fazer a coleta de informações. $O$ trabalho de campo envolve, de um lado, a organização do processo pelo staff da pesquisa-pesquisador, supervisores e entrevistadores e de outro a negociação e disponibilidade da instituição e de seus sujeitos para responderem às demandas do trabalho de campo. As pactuações iniciais são elementos fundamentais, tanto mais quanto maior for o número de sujeitos a serem disponibilizados pela instituição. Além do mais, considerando-se que a coleta de informações da pesquisa primária foi realizada em UBS, a prioridade será sempre o atendimento ao usuário.

Ainda, a negociação com a instituição torna-se, muitas vezes, um processo delicado: pode ser difícil disponibilizar seus trabalhadores para responderem perguntas sobre o seu trabalho e sobre sua saúde. Permeia a dificuldade na negociação a exposição da instituição a uma avaliação, mesmo que parcial, da sua política de recursos humanos. Este processo, muitas vezes, é contraditório, pois ao mesmo tempo que um estudo contribui com um olhar externo à identificar dificuldades, também expõe problemas e conflitos internos. Sendo assim, muitas vezes a relação é antagônica e traz tensões que podem dificultar ou impedir o trabalho de campo. São os chamados conflitos de interesse, que precisam ser previstos e explicitados nos projetos de pesquisa ${ }^{(10)}$.

Desta forma, trabalha-se em um espaço de negociação que requer do entrevistador habilidade para garantir qualidade na coleta das informações. Para tanto, este deve aprender a ser flexível e negociar espaços e disponibilidade de tempo, cedendo na medida do possível, de modo a conseguir realizar a coleta das informações. Ou seja, faz-se imprescindível aprender a trabalhar com as diversidades de interesses. Reforça-se assim que a capacitação do entrevistador, com sugestão para aumentar o seu tempo de duração, pode fazer a diferença em um processo de coleta "complicado", assegurando a continuidade e a qualidade do dado coletado.

As estratégias usadas pelos entrevistadores foram diversas e criativas, como o contato com a ASL, com a enfermeira do serviço, oferecer um agrado, mostrar-se disponível e apresentar-se bem, o contato telefônico anterior, ou a ajuda de outra entrevistadora, diminuindo o tempo de coleta na UBS. Percebeu-se, pelas respostas, que aquelas em que se oportunizou maior contato pessoal resultaram em melhores resultados, foram mais produtivas na participação dos sujeitos na entrevista.

As dificuldades encontradas na coleta das informações podem estar relacionadas com o fato de que o trabalho em saúde requer a presença humana. Assim, a utilização da técnica de entrevista, que exige interação entre pesquisador e pesquisado a fim de contextualizar as experiências, vivências e sentidos, demanda um tempo ainda maior de disponibilidade do sujeito pesquisado $^{(11)}$.

Essa situação cria duas demandas para um trabalhador, muitas vezes bastante sobrecarregado em assumir seu trabalho ${ }^{(12)} \mathrm{e}$, em casos não muito esporádicos, do seu colega que faltou, ou foi transferido, ou está de folga. Assim, se compreendem as dificuldades em coletar informações, no horário de trabalho, nesse tipo de serviço e, ao mesmo tempo, coloca para o entrevistador mais desafios em criar estratégias de convencimento à participação. São aspectos fundamentais em estudos de campo, em que os dados primários assumem um lugar central na pesquisa e, portanto, as precauções 
para diminuir os vieses de seleção não são aspectos menores nesse processo. Percebe-se, novamente, um aspecto antagônico com o trabalho de campo, pois foi afirmado pelo entrevistador, como ponto positivo, a entrevista em horário de trabalho, e, ao mesmo tempo, se depara com essas situações que precisam ser contornadas neste processo.

Pelas estratégias usadas como mais produtivas e pelas sugestões dos entrevistadores, pode-se depreender o quão árduo pode ser um trabalho de campo e o quanto de empenho pode ser necessário para efetivá-lo. Ainda, a entrada em campo pode ser destacada como fundamental para todo o processo que virá. O pesquisador, ao investir em um bom plano para entrada em campo, tende a melhorar essa etapa para os entrevistadores, assim como também o estreito acompanhamento do supervisor. Vários contatos foram feitos com a ASL, e com os enfermeiros do campo, os quais contribuíram para uma maior receptividade do entrevistador nas UBS.

As intercorrências, embora esperadas, não podem ser totalmente previstas. No caso específico da pesquisa primária, da previsão de um trabalho de campo com somente trabalhadores de um município, podem aflorar problemas de cunho coletivo e expor aspectos negativos das condições de trabalho e saúde desses trabalhadores, devendo essas intercorrências ser previstas. Talvez seja o caso, quando a proposta requeira um número considerável de trabalhadores de uma secretaria, como nesse caso, que o pesquisador planeje seu projeto de forma a não caracterizar uma prefeitura e seu setor de saúde e possa atingir seus objetivos diversificando instituições e contextos e, como decorrência, planeje a análise dessas informações levando em conta esses aspectos.

Percebe-se, pelas sugestões feitas pelos entrevistadores, que o processo de supervisão requer adequações. A ideia inicial da supervisão foi de acompanhamento de todo o processo, auxiliando o entrevistador na entrada em campo e no contato para facilitar esse processo, recebendo e distribuindo material ${ }^{(8)}$. O pagamento aos supervisores e entrevistadores deve ser uma prerrogativa de um projeto desta complexidade, que exige o acompanhamento estrito de todo o processo.

Quanto à capacitação dos entrevistadores, este estudo mostrou sua importância. Realmente a capacitação do entrevistador, peça-chave na coleta das informações, tornou-se um item sobre o qual é necessário se debruçar com muita competência quando envolve um processo complexo de organização e coleta, requerendo muitos atores e uma interligação entre eles. O entrevistador precisa estar seguro sobre como desenvolver as entrevistas, como se introduzir no campo, aspectos a serem tratados na capacitação.

Comparecer a cada campo de coleta, verificar condições e estratégias ou mesmo explicar em cada UBS, nas reuniões das equipes, sobre a pesquisa, são estratégias a serem previstas pelo staff da pesquisa.

O objeto de estudo da pesquisa primária, a saúde do trabalhador, pode expor as condições de trabalho e a linha tênue, que separa a saúde e a doença da força de trabalho. Esse objeto de estudo pode não ser uma prioridade nessas condições contextuais expostas ${ }^{(12)}$. A saúde do trabalhador é como a ponta do iceberg que só torna-se visível por meio de pesquisas como essas ${ }^{(13)}$, que mostram uma condição coletiva ${ }^{(14)}$. Fora isso, são indivíduos a adoecer e trazer transtornos aos serviços por conta das faltas, atestados e transferências para outras UBS.

Quanto ao processo de supervisão do trabalho de campo, considera-se que seja um papel novo para o pesquisador em Enfermagem, que tem realizado, majoritariamente, pesquisa qualitativa e quando realiza pesquisa quantitativa, ela tem caráter mais descritivo, 0 que simplifica em muito o processo de coleta e análise das informações, ao contrário de estudos analíticos ${ }^{(15)}$.

Assim, parece que, à medida que aumentamos a complexidade das nossas pesquisas precisaremos dispor de conhecimentos diferenciados no sentido de aprimorar o processo de coleta ${ }^{(16)}$ e análise ${ }^{(17)}$ que normalmente se aprende com outros pesquisadores mais experientes, a fim de dar suporte aos nossos objetivos e métodos. Contudo, temos este desafio à frente se quisermos avançar e propor mudanças para nossa prática profissional e de pesquisadores ${ }^{(18)}$.

\section{CONSIDERAÇÕES FINAIS}

Este estudo propôs a análise de uma etapa no processo de pesquisa: o trabalho de campo. Aos olhos de alguns, isso pode parecer estranho, ou mesmo, pouco relevante. Contudo, a experiência com a coleta de informações primárias em projetos de maior porte permite aprender a ser flexível e negociar espaços e disponibilidade de tempo, cedendo na medida do possível, de modo a conseguir realizar a coleta das informações. Ou seja, faz-se imprescindível aprender a trabalhar com as diversidades de interesses.

O consumo e a produção de conhecimento são atividades inerentes ao exercício profissional e exigem habilidades e conhecimentos específicos, que podem ser aperfeiçoados em grupos de pesquisa, no convívio 
com outros pesquisadores e por meio de publicações. Do mesmo modo, a pesquisa metodológica tem sua importância à medida que contribui para o aperfeiçoamento dos procedimentos realizados, diminuindo vieses, que tão fortemente influenciam os resultados em pesquisa, o seu rigor e aplicabilidade. A pesquisa é uma tarefa de todos nós e para a qual temos que buscar e construir conhecimento. Acreditamos que a qualidade do processo de pesquisa, o uso adequado e assertivo do método, incluindo-se aí os procedimentos necessários à diminuição de perdas e recusas é, condição sine qua non para se chegar à qualidade de pesquisa em saúde.

\section{REFERÊNCIAS}

1. Bittar DB, Pereira LV, Lemos RCA. Sistematização da assistência de Enfermagem ao paciente crítico: proposta de instrumento de coleta de dados. Texto Contexto Enferm. 2006;15(4):617-28.

2. Galdeano LE, Rossi LA. Construção e validação de instrumentos de coleta de dados para o período perioperatório de cirurgia cardíaca. Rev. Latino-Am. Enfermagem 2002;10(6):800-4.

3. Yonekura T, Soares CB. The educative game as a sensitization strategy for the collection of data with adolescents. Rev. Latino-Am. Enfermagem 2010;18(5):968-74.

4. Cibreiros AS, Oliveira ICS. A dramatização no espaço hospitalar: uma estratégia de pesquisa com crianças. Esc. Anna Nery. 2010;14(1):165-70.

5. Santos CS, Cerchiari EAN, Alvarenga MRM, Faccenda O, Oliveira MAC. Avaliação da confiabilidade do mini-exame do estado mental em idosos e associação com variáveis sociodemográficas. Cogitare enferm. 2010;15(3):406-12.

6. Pessalacia JDR, Ribeiro CRO. Vulnerabilidade e riscos em pesquisas com entrevista. Nursing (São Paulo). 2007;10(114):509-13.

7. Martins CC, Ramos-Jorge ML, Cury JÁ, Pordeus IA, Paiva SM. Agreement between data obtained from repeated interviews with a six-years interval. Rev. Saúde Pública. 2008;42(2): 346-9.

8. Camponogara S, Kirchhof ALC, Gelbcke FL, Magnago TSBS. O espaço do diálogo na pesquisa em enfermagem: relato de experiência sobre a fase de coleta de dados. Texto Contexto Enferm. 2007;16(4):762-8.
9. Minayo, M.C.S. O desafio do conhecimento: pesquisa qualitativa em saúde. 12a ed. São Paulo: HucitecAbrasco, 2010.

10. FAPESP. Fundação de Amparo a Pesquisa do Estado de São Paulo. Código de boas práticas científicas.[acesso em 10 nov 2011] 2012. Disponível: http://www.fapesp. br/boaspraticas/codigo_050911.pdf

11. Silva GRF, Macedo KNF, Rebouças CBA, Souza AMA. Interview as a technique of qualitative research - a literature review. Online braz. j. nurs. [Internet] 2009;5(2) [acesso em 10 nov 2011]. Disponível: http:// www.objnursing.uff.br/index.php/nursing/article/ view/382/88

12. Silva LAA, Ferraz F, Lino MM, Backes VMS, Schimidt SMS. Educação permanente em saúde e no trabalho de enfermagem: perspectiva de uma práxis transformadora. Rev. Gaúcha Enferm. 2010;31(3):557-61.

13. Guevara EB, Mendias EP. A comparative analysis of the changes in nursing practice related to health sector reform in five countries of the Americas. Rev. panam. salud publica. 2002;12(5):347-53.

14. Silveira DT, Marin HF. Conjunto de dados mínimos de enfermagem: construindo um modelo em saúde ocupacional. Acta Paul. Enferm. 2006;19(2):218-27.

15. Lima-Costa MF, Barreto MS. Tipos de estudos epidemiológicos: conceitos básicos e aplicação na área do envelhecimento. Epidemiol. Serv. Saude. 2003;12(4):189-201.

16. Barros AJD, Hirakata VN. Alternatives for logistic regression in cross-sectional studies: an empirical comparison of models that directly estimate the prevalence ratio. BMC medical research methodology 2003;3(21) DOI: 10.1186/1471-2288-3-21.

17. Marin HF, Barbieri M, Barros SMO. Conjunto internacional de dados essenciais de enfermagem: comparação com dados na área de saúde da mulher. Acta Paul. Enferm. 2010;23(2):251-6.

18. Lunardi VL. Qual deve ser o objetivo de investigações a serem posteriormente recomendadas pelo comitê assessor da área de enfermagem no CNPQ. Cienc. cuid. saude. [Internet] 2009;8(3) [acesso em 10 nov 2011]. Disponível: http://periodicos.uem.br/ojs/index.php/ CiencCuidSaude/article/view/11034/5933 